\title{
Double-diffusive flow in a porous right-angle trapezoidal enclosure with constant heat flux
}

\author{
Zafar Khan ${ }^{1}$, W.A. Khan ${ }^{2}$, Muhammad Qasim ${ }^{3}$, and Min Du ${ }^{1}$ \\ ${ }^{1}$ Sichuan University \\ ${ }^{2}$ Prince Mohammad Bin Fahd University \\ ${ }^{3}$ COMSATS Institute of Information Technology
}

October 21, 2020

\begin{abstract}
A computation analysis is performed to study double-diffusive natural convection in a right-angle trapezoidal cavity packed with a porous medium. The horizontal top and bottom boundaries are insulated and impermeable. The vertical left sidewall is kept at a constant heat flux and high concentration, whereas the temperature and concentration of the mixture at the inclined sidewall are held at lower temperatures and lower concentration. The dimensionless nonlinear system is solved by employing finite difference method along Successive Under Relaxation technique. The findings are compared and validated with the existing literature for the Darcy flow driven through a single buoyancy effect (difference in density is only due to temperature variations) in a porous square enclosure. The numerical results are expressed in the form of dimensionless temperature, concentration, streamlines, isotherms and iso-concentrations, local and average Nusselt and Sherwood numbers. It is demonstrated that the Rayleigh number and buoyancy parameter enhance both surface heat and concentration rates.
\end{abstract}

\section{Hosted file}

Manuscript.pdf available at https://authorea.com/users/369215/articles/488141-doublediffusive-flow-in-a-porous-right-angle-trapezoidal-enclosure-with-constant-heat-flux 
figures/Figure1/Figure1-eps-converted-to.pdf 
figures/Figure2/Figure2-eps-converted-to.pdf 
figures/Figure3(anb)/Figure3(anb)-eps-converted-to.pdf 
figures/Figure3(c)/Figure3(c)-eps-converted-to.pdf 
figures/Figure4/Figure4-eps-converted-to.pdf 
figures/Figure5/Figure5-eps-converted-to.pdf 
figures/Figure6/Figure6-eps-converted-to.pdf 
figures/Figure7/Figure7-eps-converted-to.pdf 
figures/Figure8/Figure8-eps-converted-to.pdf 
figures/Figure9/Figure9-eps-converted-to.pdf 